\title{
RAPID DETECTION OF VITAMIN C CONTENT IN FRUITS AND VEGETABLES USING A DIGITAL CAMERA AND COLOR REACTION
}

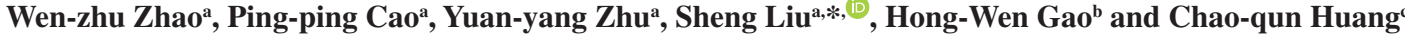 \\ ${ }^{a}$ College of Computer Science and Technology, Huaibei Normal University, Huaibei 235000, P.R. China \\ ${ }^{b}$ College of Environmental Science and Engineering, Tongji University, Shanghai 200092, P.R. China \\ 'Hefei Institutes of Physical Science, Chinese Academy of Science, Hefei 230031, P.R. China
}

Recebido em 25/05/2020; aceito em 10/08/2020; publicado na web em 21/09/2020

\begin{abstract}
In this study, we propose a novel method to determine the vitamin $\mathrm{C}$ content in fruits and vegetables. The proposed method exploits the image and data processing abilities of computers by coupling a digital camera and a color reaction. An image acquisition device was developed to collect the image information of the solution to be measured after the color reaction. The corresponding RGB and Lab color components were obtained by an image analysis software. Further, the standard curves of the vitamin $\mathrm{C}$ concentration and color component were established by nonlinear, linear, and multivariate data fitting. The detection range was $0.81-20.00 \mu \mathrm{g} \mathrm{mL}$ $\left(\mathrm{R}^{2}=0.9994\right)$, LOD was $0.24 \mu \mathrm{g} \mathrm{mL}{ }^{-1}$, LOQ was $0.81 \mu \mathrm{g} \mathrm{mL} \mathrm{m}^{-1}$, accuracy was $98.95 \%$, and precision was determined with an RSD of $0.73 \%$. A comparison of the results confirmed that the proposed method's accuracy was higher than that of the spectrophotometric method. The proposed method has a simple design and facilitates easy operation, and the cost of the equipment is only US\$31.43. Therefore, it can replace the traditional spectrophotometric method for the determination of vitamin $\mathrm{C}$ content in fruits and vegetables and is highly applicable in the food, chemical, and medical fields.
\end{abstract}

Keywords: measurement technique; vitamin C content; digital camera; image processing; multivariate fitting.

\section{INTRODUCTION}

Vitamin C, also known as ascorbic acid, is a water-soluble vitamin with strong antioxidant activity. It can optimize cell and tissue levels to prevent and treat respiratory and systemic infections. ${ }^{1}$ Vitamin C deficiency results in impaired immunity and higher susceptibility to infections. A high dose of vitamin $\mathrm{C}$ can be used as an inexpensive, efficient, and multifaceted antioxidant, which can prevent or restore microcirculation disorders, protect against oxidative stress induced by ischemia, sepsis, or burns, and alleviate organ damage and dysfunction. $^{2}$

Vitamin $\mathrm{C}$ is synthesized by a variety of organisms of the animal and plant kingdoms. However, humans, other primate animals, and guinea pigs cannot produce this vitamin. ${ }^{3}$ Consequently, when humans do not ingest vitamin $\mathrm{C}$ through their diets, a deficiency state occurs with a wide spectrum of clinical manifestations. Clinical expression of vitamin $\mathrm{C}$ deficiency, i.e., scurvy, is a lethal condition unless appropriately treated. Thus, humans must ingest vitamin $\mathrm{C}$ to survive. ${ }^{4}$ A very small daily intake of vitamin C (10-15 mg/day for an adult) is required to avoid deficiency and scurvy. ${ }^{5}$ However, excessive intake of vitamin $\mathrm{C}$ can lead to secondary oxalidosis, resulting in kidney damage. ${ }^{6}$ Further, excessive intake of vitamin $\mathrm{C}$ may induce obesity. ${ }^{7}$ Therefore, to effectively control the daily intake of vitamin $\mathrm{C}$, the quantitative determination of its content in fruits and vegetables is crucial.

At present, the main methods for the determination of vitamin C content include titration, spectrophotometry, ${ }^{8}$ fluorometry, ${ }^{9}$ highperformance liquid chromatography (HPLC), ${ }^{10}$ and electrochemical analysis. ${ }^{11}$ The titration methods mainly include direct iodometry ${ }^{12}$ and the 2,6-dichloroindophenol method. ${ }^{13}$

The iodometric method uses starch as an indicator and an iodine standard solution to titrate the vitamin $\mathrm{C}$ solution. When vitamin $\mathrm{C}$ is completely oxidized, the excessive iodine will react with the

*e-mail: liurise@139.com starch causing the solution to turn blue and indicating the end point of titration. Depending on the amount of iodine consumed in the experiment, the content of vitamin $\mathrm{C}$ in the tested sample can be calculated. In the second method, 2,6-dichloroindophenol is used as an indicator. The redox titration of the vitamin $\mathrm{C}$ solution is conducted using a blue 2,6-dichloroindophenol solution, which is subsequently reduced to a colorless solution by vitamin $\mathrm{C}$. When the end point of titration is reached, the redundant 2,6-dichloroindophenol appears as light red in an acidic medium. The content of vitamin $\mathrm{C}$ in the solution is calculated from the consumption of 2,6-dichloroindophenol. The method is convenient and fast, but the reaction process is easily disturbed by other reducing substances; therefore, the measurement accuracy is low. ${ }^{14}$

The advantages of the spectrophotometry, fluorescence, and HPLC methods include high sensitivity, good selectivity, rapid determination, and no influence of color. However, the required equipment is expensive and bulky, which is unsuitable for people without laboratory training. Commercial spectrophotometers are expensive and have low sensitivity and specificity. Therefore, several other substances may interfere with the chromogen or enzyme used and reduce the accuracy of the measurement. ${ }^{15,16}$ The procedure and instrumentation of the electrochemical method are relatively simple; the sample pretreatment requirements are low, the response is fast, and the method is cost efficient. The amperometric method also exhibits the above-mentioned characteristics and has high sensitivity; however, the complex medium may easily interfere with the electrochemical method during vitamin $\mathrm{C}$ content determination. ${ }^{17,18}$

In recent years, advances in computer vision, open source technology, and other technical fields have enabled the development of new analytical methods based on digital images that can be widely used in measurement applications. These methods are often referred to as digital image based (DIB or DIA) methods. The DIB method usually uses images captured from colorimetric or luminescent reactions promoted by a reagent and an analyte with selective interaction to determine the concentration of the analyte in a sample. ${ }^{19}$ 
This noninvasive, low-cost method features a wide dynamic range, high sensitivity, and low waste generation during experiments, which conform to the principle of green chemistry. Owing to these favorable characteristics, the DIB method has been applied to various scientific fields. For example, in the field of biology, a DIB-based method was developed to determine the total titratable acidity of orange, lemon, and passion fruit based on a spot test obtained from digital images and use of anthocyanins as the biodegradable indicator. ${ }^{19}$ In the field of chemistry, the reflected radiation intensity of digital images was collected through smartphone cameras to determine the content of methanol in biodiesel. ${ }^{20}$ This technique was developed based on the discoloration of acidic potassium permanganate solution and methanol oxidation. Using a web camera instead of a colorimetric sensor, the image processing technology of the RGB and HSV color systems is combined with various electrochemical technologies to be applied to a colorimetric detection system. ${ }^{21}$ In soil analysis, the RGB values of digital images and the derived soil indexes are used to determine the content of trinitrotoluene (TNT) in soil. ${ }^{22}$

Based on the DIB method, Coutinho et al. conducted smartphone image analysis for the quantification of vitamin $\mathrm{C}$ based on its interfering effect in the enzymatic colorimetric detection of glucose. ${ }^{23}$ Additionally, dos Santos et al. employed a smartphone to capture images obtained from a colorimetric spot test to determine the vitamin $\mathrm{C}$ content in Brazilian Amazon native and exotic fruits. ${ }^{24}$

In this study, we propose a method for determining the vitamin $\mathrm{C}$ content in fruits and vegetables using a commercially available digital camera and deploying the color reaction of colored compounds. The use of an image acquisition device and an image processing software to determine the vitamin $\mathrm{C}$ content (i.e., a DIB-based technique) facilitates a green, simple, and efficient method for the determination of the vitamin $\mathrm{C}$ content.

Digital cameras mostly use CMOS photosensitive elements, which are semiconductor elements used to record light changes. They are mainly composed of silicon and germanium. The advantages of semiconductor elements include high integration, low power consumption, and low cost. Each pixel of the camera is equivalent to a photoelectric detection element, and the signal processing unit integrated by the camera can replace the digital-toanalog conversion circuit and the signal processing circuit of the spectrophotometer.

The measurement method in this study is based on the reducibility of vitamin C. Vitamin $\mathrm{C}$ reduces $\mathrm{Fe}$ (III) to $\mathrm{Fe}$ (II) and then forms a complex with o-phenanthroline. The strong red complex of $\mathrm{Fe}$ (II) and o-phenanthroline is widely used for colorimetric determination and as an oxidation-reduction indicator. ${ }^{25}$ This reaction is characterized by high speed, stability, and reproducibility, and the reaction waste is simple to treat. ${ }^{26}$ Therefore, o-phenanthroline was selected as the chromogenic agent to determine the vitamin $\mathrm{C}$ content. A schematic representation for the detection of vitamin $\mathrm{C}$ is shown in Figure 1. The reaction is rapid and stable and is not disturbed by amino acids, citric acid, carbohydrates, starch, or common metal ions. Therefore, the use of a camera coupled with the color reaction of colored compounds improves the accuracy and reduces the cost of measuring the vitamin $\mathrm{C}$ content.

\section{EXPERIMENTAL}

\section{Design of experimental device}

\section{Device structure}

The experimental system consists of the solution image acquisition device and image analysis software. The configuration of the system is shown in Figure 2(a).

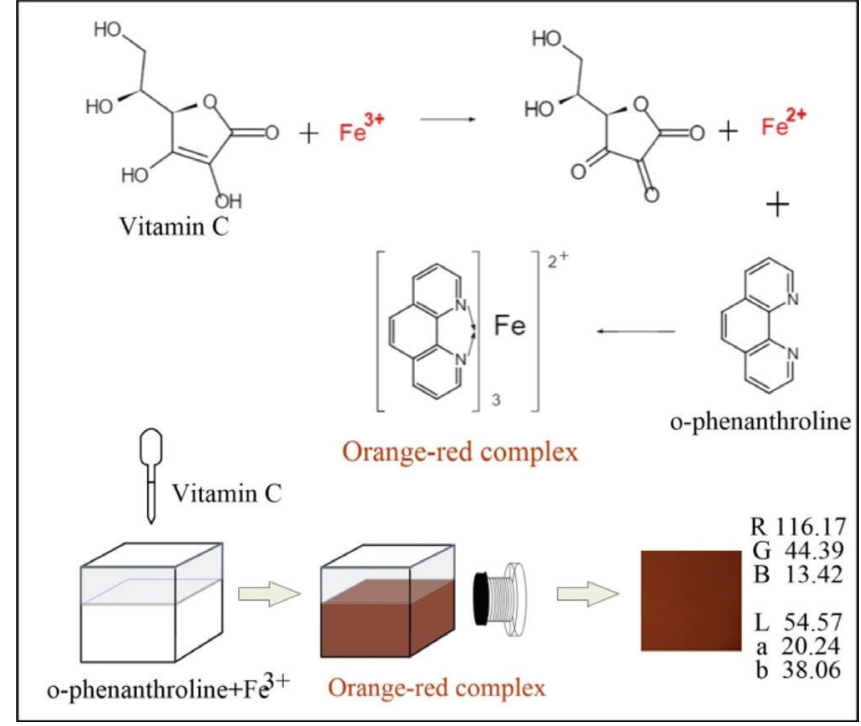

Figure 1. Schematic representation for the detection of vitamin $C$ by using o-phenanthroline

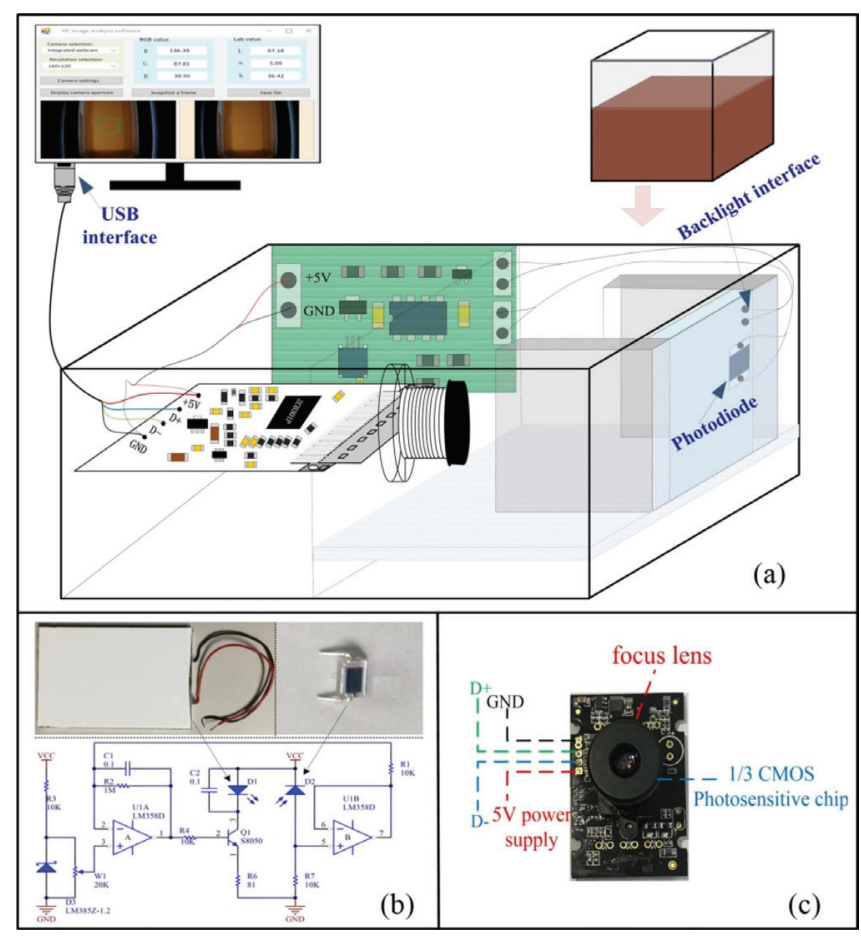

Figure 2. Configuration of the vitamin C content measuring setup: (a) measurement device, (b) constant current circuit for the light source, (c) digital camera

The image acquisition device consists of a constant light intensity circuit, backlight plate, sample reservoir, and a common digital camera. The outer shell of the device is bound to a black acrylic board and carries a black plastic cover to seal off the sampling area without interference from the external light source. The light source system uses a backlight plate to make the light more uniform, and the light source is driven by a constant light intensity circuit to make the light more stable. The backlight board consists of a light guide, brightener, reflector, and two 0.3 -W white LEDs (color temperature of 5,700 K). The backlight plate is tightly connected to the sampling unit. When the backlight plate emits white light, the light passes through the chromogenic solution to the camera; the solution reflects and transmits the light of its own wavelength and absorbs the light of other wavelengths. Thus, the camera 
obtains the image of the solution after color rendering, which contains information on the vitamin $\mathrm{C}$ concentration.

\section{Transmission and reflection method}

The two modes of reflection and transmission are the basic means of spectrum measurement. The reflection light method refers to the reflection of light from the object surface, while the transmission light method refers to the collection of transmitted light after passing through the sample. Compared with direct reflection, transmission is less interfered by ambient light. When the reflection method is used to measure the concentration of medium analyte, the distribution of the path length of light propagating in the medium before leaving the incident side is relatively wide, resulting in a significant decrease in the quantitative accuracy of measuring the concentration of medium analyte. ${ }^{27}$ This study implements measurement by the transmission light method, which can reflect the solution characteristics more accurately. Moreover, the proposed method uses a device with a fixed optical path, which reduces the impact of environmental factors on image acquisition.

\section{Image acquisition and processing software}

The image analysis software was designed based on a VS2012 development environment, using the $\mathrm{C \#} \mathrm{language} \mathrm{and} \mathrm{Camera-}$ NET (.NET library for easy use of camera via DirectShow). The acquisition program collects camera data through a USB interface and processes the data to obtain the corresponding color component value of the solution.

The backlight plate and driver circuit are shown in Figure 2(b). The backlight plate has a uniform light source and good imaging quality. The circuit is powered by a USB. Diode D2 collects the light intensity of the LED and controls the working current of the backlight plate so that the intensity of the light source is constant. The working current of LED can be changed by adjusting W1. In the experiment, the working current of LED is $12 \mathrm{~mA}$. This is a closed-loop control circuit, which is relatively simple, but the effect is better than that of having constant current.

To ensure the accuracy of the experiment, the camera should have uniform parameters during calibration and measurement. Therefore, the automatic functioning of the camera must be disabled, allowing for manual adjustment of the focal length, exposure, and white balance functions to ensure consistent parameter values throughout the measurement process. The model of the camera used in this study is JD300, which can perform image processing, image compression, and USB data transmission functions. The camera uses a CMOS sensor, and its structure is shown in Figure 2(c).

The software interface is shown in Figure 1S, and the operating environment is a personal computer running the Windows 10 operating system. The main function of the software is to acquire the camera image and obtain the required color components after processing. As shown in Figure 1S(a), the camera parameters are fixed when the solution image is acquired to ensure consistency throughout the measurement process. When extracting the RGB color value of the image, only 400 pixels in the central region of the image are extracted, which avoids the brightness interference in the surrounding area of the image. Then, the average value of RGB is calculated, and the corresponding CIELab values are obtained by the conversion from RGB color space to Lab color space. In addition, the position and focal length of the camera need to be adjusted to obtain a clear solution image. The software interface and key function are shown in Figure 1S(b). The interface contains the RGB and CIELab values of the solution image.

Vitamin C reacts with Fe (III) and o-phenanthroline in weak acid conditions to form orange-red compounds. The image of the orange-red liquid is captured by an ordinary digital camera (JD300, Shenzhen, China), and the average RGB value of the image central region is obtained. Then, the RGB values are transformed into $\mathrm{XYZ}$ values by changing the color space; then, the XYZ values are transformed into the corresponding experimental CIELab values..$^{28,29}$

First, the RGB values are converted to XYZ values by using the following formulas:

$$
\left[\begin{array}{l}
X \\
Y \\
Z
\end{array}\right]=\left[\begin{array}{lll}
0.412453 & 0.357580 & 0.180423 \\
0.213671 & 0.715160 & 0.072169 \\
0.019334 & 0.119193 & 0.950227
\end{array}\right]\left[\begin{array}{l}
R \\
G \\
B
\end{array}\right]
$$

The XYZ values are obtained by applying the conversion matrix of the sRGB standard, which is an international standard for color space defined by the International Electrotechnical Commission (IEC) (IEC 61966-2-1: IEC 1999). ${ }^{30}$

$$
\left\{\begin{array}{l}
X=\frac{X}{255 \times 0.950456} \\
Y=\frac{Y}{255} \\
Z=\frac{Z}{255 \times 1.088754}
\end{array}\right.
$$

Then, the XYZ values are transformed into the CIELab values:

$$
\begin{gathered}
\left\{\begin{array}{l}
L=116 f\left(\frac{Y}{100.0}\right)-16 \\
A=500\left[f\left(\frac{X}{95.047}\right)-f\left(\frac{Y}{100.0}\right)\right] \\
B=500\left[f\left(\frac{Y}{100.0}\right)-f\left(\frac{Z}{108.883}\right)\right]
\end{array}\right. \\
f(t)= \begin{cases}t^{\frac{1}{3}} & \text { if } t>\left(\frac{6}{29}\right)^{3} \\
\frac{1}{3}\left(\frac{29}{6}\right) t^{2}+\frac{4}{29} & \text { otherwise }\end{cases}
\end{gathered}
$$

The RGB color space uses the three primary colors to represent colors. The Lab color space consists of one brightness channel and two-color channels. L represents brightness, a represents the greento-red component, and b represents the blue-to-yellow component. Compared with the RGB color space, the Lab color space is uniformly perceived and device independent. The experimental data of different vitamin $\mathrm{C}$ solutions and corresponding color components were obtained. Different fitting methods were used to fit the relationship between concentration and corresponding RGB and CIELab values. The fitting degree of different methods was compared to determine the best fitting method. Finally, the standard curve for the determination of the actual vitamin $\mathrm{C}$ content was obtained.

\section{Preparation of standard solution and data acquisition}

\section{Reagents}

In this experiment, ascorbic acid, glacial acetic acid, sodium acetate trihydrate, sodium hydroxide titration solution $\left(0.5 \mathrm{~mol} \mathrm{~L}^{-1}\right)$, oxalic acid dihydrate, and kaolin were used. They were purchased from Sinopharm Chemical Reagent Co., Ltd. (https://www.reagent. com.cn, Shanghai, China). O-phenanthroline ferric ammonium sulfate was purchased from Xilong Scientific Co., Ltd. (http://www. 
xlhg.com.cn, Shantou, China). All chemicals were of analytical grade and were used directly without further purification. Deionized water obtained from Shanghai Jingchun Water Technologies Co., Ltd. (resistance 10-18 M $\Omega \mathrm{cm}^{-1}$; http://www.shkys.com, Shanghai, China) was used throughout the experiments.

Different concentrations of the standard vitamin C solution were prepared with a standard solution of $2.5 \mathrm{mg} \mathrm{mL}^{-1}$. The o-phenanthrene solution $\left(4.0 \mathrm{mg} \mathrm{mL}^{-1}\right.$ ) was used as a chromogenic reagent in the chromogenic reaction. The ferric ammonium sulfate solution (2.4 $\mathrm{mg} \mathrm{mL}^{-1}$ ) was used to provide the Fe (III) ion. The buffer solution ( $\mathrm{pH} 4-5)$ was prepared to provide an acidic environment. Oxalic acid dihydrate $(2.5 \mathrm{~g})$ was dissolved in $100 \mathrm{~mL}$ of deionized water, and then $250 \mathrm{~mL}$ of deionized water was added to the solution. The final $1 \%$ oxalic acid solution was prepared to extract vitamin $\mathrm{C}$ from fruit samples.

\section{Calibration procedure}

To ensure the repeatability of the experiment, we took a standard solution of 0 point and intermediate concentration value to calibrate the experimental device before each experiment. At 0 point, we used the blank solution without adding the vitamin $\mathrm{C}$ solution. We added $0.6 \mathrm{~mL}$ of a $4.0 \mathrm{mg} \mathrm{mL}^{-1}$ o-phenanthrene solution and $0.6 \mathrm{~mL}$ of a $2.4 \mathrm{mg} \mathrm{mL}^{-1}$ ammonium ferric sulfate solution into the $15 \mathrm{~mL}$ test tube. Finally, we added the sodium acetate buffer solution to achieve a total volume of $10 \mathrm{~mL}$. The standard solution with a vitamin $\mathrm{C}$ concentration of $10 \mu \mathrm{g} \mathrm{mL}^{-1}$ was used for the middle scale. After the instrument was stabilized, we adjusted the backlight intensity (by adjusting the $\mathrm{W} 1$ on the backlight plate) and acquired the image by adjusting the camera parameters.

If the method is used as a complete instrument, the calibration function of the software should be designed according to the analytical formula used in the measurement; i.e., the coefficient of the analytical formula can be obtained through the 0 point and the range point for the measurement of a vitamin $\mathrm{C}$ solution of unknown concentration.

\section{Standard vitamin $C$ solution and corresponding color components}

The standard solution of vitamin $\mathrm{C}$ was prepared by first adding 2 , $4,12,20,28,36,40,44,52,60,68,76$, and $80 \mathrm{~mL}$ of the $2.5 \mathrm{mg} \mathrm{mL}^{-1}$ standard solution of vitamin $\mathrm{C}$ in a $15 \mathrm{~mL}$ test tube. Then, $0.6 \mathrm{~mL}$ of the $4.0 \mathrm{mg} \mathrm{mL}^{-1} \mathrm{o}$-phenanthrene solution and $0.6 \mathrm{~mL}$ of thee $2.4 \mathrm{mg} \mathrm{mL}^{-1}$ ferric ammonium sulfate solution were sequentially added. Finally, sodium acetate buffer solution was added to bring the total volume to $10 \mathrm{~mL}$. In this way, $0.5,1,3,5,7,9,10,11,13$, $15,17,19$, and $20 \mu \mathrm{g} \mathrm{mL} \mathrm{m}^{-1}$ standard solutions were prepared. The blank solution in the experiment, which was used as a reference, was prepared by using the same dosage of o-phenanthrene solution and ferric ammonium sulfate solution.

After the standard solution was stabilized, it was placed in a colorimetric dish. Then, the colorimetric dish was placed in the image acquisition device, and the RGB and CIELab values of the solution were obtained by the image processing software. The obtained color and color components are shown in Figure 3.

With the increase in the concentration of vitamin $\mathrm{C}$ in the standard solution, the content of $\mathrm{Fe}$ (II) formed by the quantitative reduction of vitamin C and Fe (III) increased. Simultaneously, the orangered complex formed by the reaction of Fe (II) and o-phenanthrene gradually increased, and the corresponding solution image color gradually deepened. The color information of the solution contains the information of vitamin $\mathrm{C}$ concentration.

The image processing software obtained the corresponding RGB values of the image and converted it into the corresponding Lab values. The color component data obtained are shown in Table 1 . To verify the correctness of the color value conversion, the results obtained were compared with the conversion results of the Adobe Photoshop CC 2019 software.

The Lab values converted by the image processing software were compared with the Lab values converted by the Adobe Photoshop CC 2019 software, and the results were found to be identical. The RGB values and brightness value $L$ of the image gradually decreased, while the $\mathrm{a}$ and $\mathrm{b}$ values generally increased but also fluctuated. Therefore, the vitamin $\mathrm{C}$ concentration can be determined by the change of color component.

\section{RESULTS AND DISCUSSION}

\section{Data fitting results}

\section{Nonlinear fitting results}

The widely used linear regression model may not guarantee accuracy when used in practice because of certain limitations of the data. Furthermore, the data collected by low precision systems often have nonlinear characteristics. ${ }^{31}$ Therefore, an increasing number of nonlinear models have been applied to the quantitative analysis of concentration. Ma et al. determined the concentration of blood
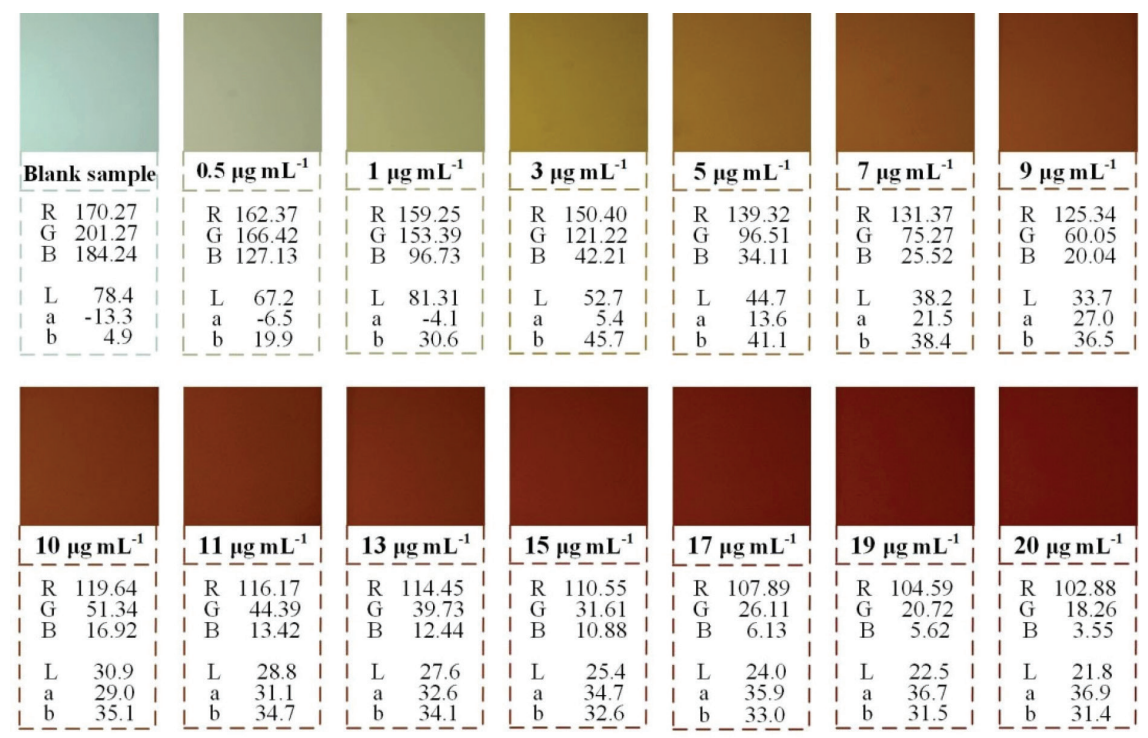

Figure 3. Color and color components of the standard vitamin C solutions 
Table 1. Vitamin C standard solution image color component

\begin{tabular}{lcccccc}
\hline Vitamin $\mathrm{C}$ concen- $_{\text {tration }\left(\mu \mathrm{mL}^{-1}\right)}$ & \multicolumn{6}{c}{ Color component } \\
\cline { 2 - 7 } & $\mathrm{R}$ & $\mathrm{G}$ & $\mathrm{B}$ & $\mathrm{L}$ & $\mathrm{a}$ & $\mathrm{b}$ \\
\hline 0 & 170.27 & 201.27 & 184.24 & 78.4 & -13.3 & 4.9 \\
0.5 & 162.37 & 166.42 & 127.13 & 67.2 & -6.5 & 19.9 \\
1 & 159.25 & 153.39 & 96.73 & 62.9 & -4.1 & 30.6 \\
3 & 150.40 & 121.22 & 42.21 & 52.7 & 5.4 & 45.7 \\
5 & 139.32 & 96.51 & 34.11 & 44.7 & 13.6 & 41.1 \\
7 & 131.37 & 75.27 & 25.52 & 38.2 & 21.5 & 38.4 \\
9 & 125.34 & 60.05 & 20.04 & 33.7 & 27.0 & 36.5 \\
10 & 119.64 & 51.34 & 16.92 & 30.9 & 29.0 & 35.1 \\
11 & 116.17 & 44.39 & 13.42 & 28.8 & 31.1 & 34.7 \\
13 & 114.45 & 39.73 & 12.44 & 27.6 & 32.6 & 34.1 \\
15 & 110.55 & 31.61 & 10.88 & 25.4 & 34.7 & 32.6 \\
17 & 107.89 & 26.11 & 6.13 & 24.0 & 35.9 & 33.0 \\
19 & 104.59 & 20.72 & 5.62 & 22.5 & 36.7 & 31.5 \\
20 & 102.88 & 18.26 & 3.55 & 21.8 & 36.9 & 31.4 \\
\hline
\end{tabular}

components by building a nonlinear regression method. ${ }^{32}$ Munmai and Sombook used the approach of combining spectrophotometry with a nonlinear method to determine the $\mathrm{pKa}$ value of a red cabbage solution. ${ }^{33}$

By measuring the RGB and CIELab values of standard vitamin $\mathrm{C}$ solutions with different concentrations, we were able to fit the concentration using the RGB and Lab values. The results are shown in Figure 4, and the expressions of the fitting results are shown in Table 2.

The values of $\mathrm{R}, \mathrm{G}, \mathrm{B}, \mathrm{L}, \mathrm{a}$, and $\mathrm{b}$ were all nonlinear with the concentration of vitamin $\mathrm{C}$. The correlation coefficient of the fitting curve between the value of $\mathrm{R}$ and vitamin $\mathrm{C}$ concentration is 0.9961 using the exponential fitting method, which is the highest correlation coefficient between the $\mathrm{R}$ value and vitamin $\mathrm{C}$ concentration.

Table 2. Fitting expressions of the R, G, B, L, a, and b values for the vitamin $\mathrm{C}$ concentration

\begin{tabular}{ccc}
\hline Color components & Expression & Adj. $\mathrm{R}^{2}$ \\
\hline $\mathrm{R}$ & $y=75.328 e^{-\mathrm{x} / 10.628}+92.659$ & 0.9961 \\
$\mathrm{G}$ & $y=174.469 e^{-\mathrm{x} / 6.625}+12.505$ & 0.9911 \\
$\mathrm{~B}$ & $y=163.948 e^{-\mathrm{x} / 1.623}+13.491$ & 0.9819 \\
$\mathrm{~L}$ & $y=51.578 e^{-\mathrm{x} / 11.436}+36.647$ & 0.9952 \\
$\mathrm{a}$ & $y=-0.019 \mathrm{x} 2+2.601 \mathrm{x}-7.909$ & 0.9925 \\
$\mathrm{~b}$ & $y=-37.277 e^{-x / 1.376}+38.676$ & 0.9644 \\
\hline
\end{tabular}

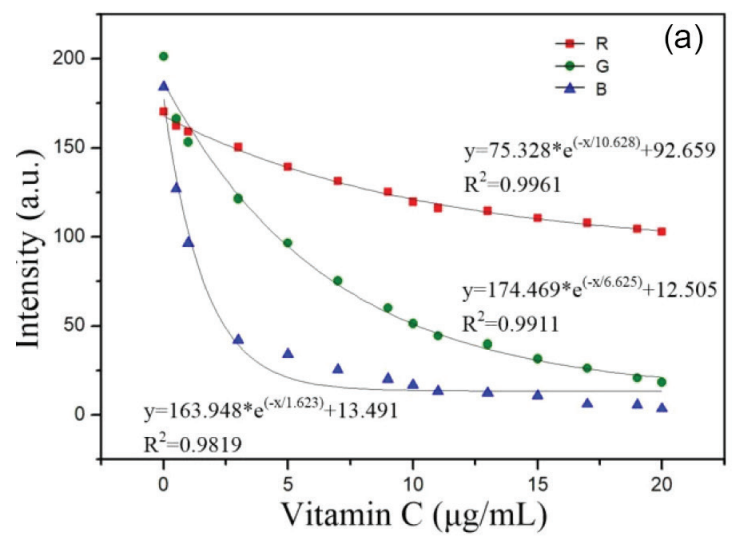

\section{Linear fitting results}

To obtain the linear relation curve, we needed to further process the color component. The RGB and $\mathrm{L}$ values corresponding to the collected solution image were processed by calculating the absorbance, according to equation (5):

$$
\mathrm{A}_{k}=-\log \frac{I_{k}}{I_{0, k}}
$$

where $A_{k}$ is the absorbance of a given channel $k$ (red, green, blue, or brightness); $\mathrm{I}_{\mathrm{k}}$ is the intensity of a given channel k; and $\mathrm{I}_{0, \mathrm{k}}$ is the blank intensity of a given channel $\mathrm{k}$.

Building the fitting curve between the obtained absorbance value and vitamin $\mathrm{C}$ concentration (see Figure 5), we find that the calibration curve of the color component and vitamin $\mathrm{C}$ concentration is linear. Among the RGB and $\mathrm{L}$ values, the linear regression constructed using the G-resolved absorbance value is $y=0.0484 x+0.7771$, and the correlation coefficient $\mathrm{R}^{2}$ reaches 0.9983 .

The results are consistent with the literature, which reports that the red, green, and blue channels significantly differ. The color of the red channel is close to that of the solution, making the results of the red absorbance relatively ideal. In addition, the trend of red absorbance is consistent with the brightness L, mainly because the absorption of the solution to red is consistent with the attenuation of brightness. In the green response, there is an artifact at the peak value close to $490 \mathrm{~nm}$, possibly caused by the cross talk between green and blue pixels, which makes the $G$ value quite different from the other channels. The green, red, and blue channels have a large cross in the spectral sensitivity curves. ${ }^{34}$ However, from the perspective of absorbance, the red channel and $\mathrm{L}$ channel have the best results.

The RGB color components of the vitamin $\mathrm{C}$ reaction solution were also converted to a grayscale value using formula (6).

$$
\text { grayscale }=R \times 0.299+G \times 0.587+B \times 0.114
$$

This formula is the standard algorithm used by image processing software (e.g., GIMP). It is implemented by MATLAB's "rgb2gray" function, and it is frequently used in computer vision. ${ }^{35}$ Compared with the direct operation on a color image, using a grayscale image simplifies the algorithm and reduces the amount of calculation. The benefits of RGB systems in many applications may be limited, and the introduction of unnecessary information may increase the amount of training data needed to achieve good performance. ${ }^{36}$ The grayscale and log-grayscale values can also be used as analytical signals for the determination of vitamin $\mathrm{C}$ concentration, as shown in Figure 2S.

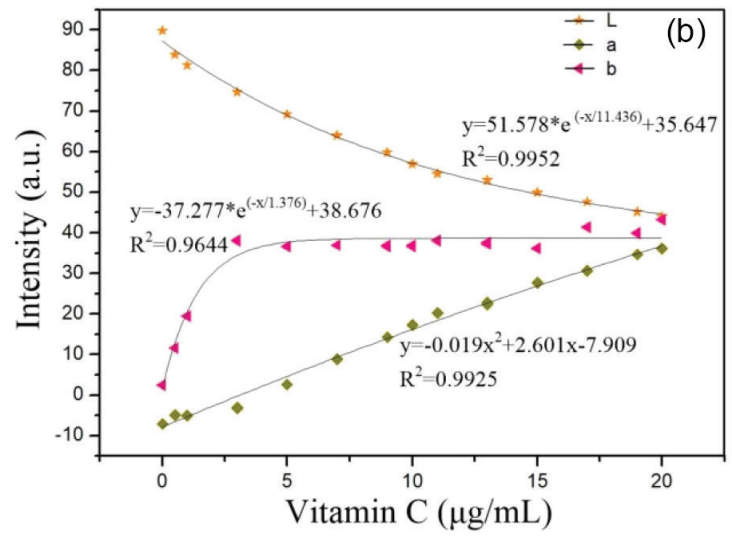

Figure 4. Fitting results of vitamin C concentration and corresponding color components: (a) vitamin C fitted with RGB, (b) vitamin C fitted with Lab 

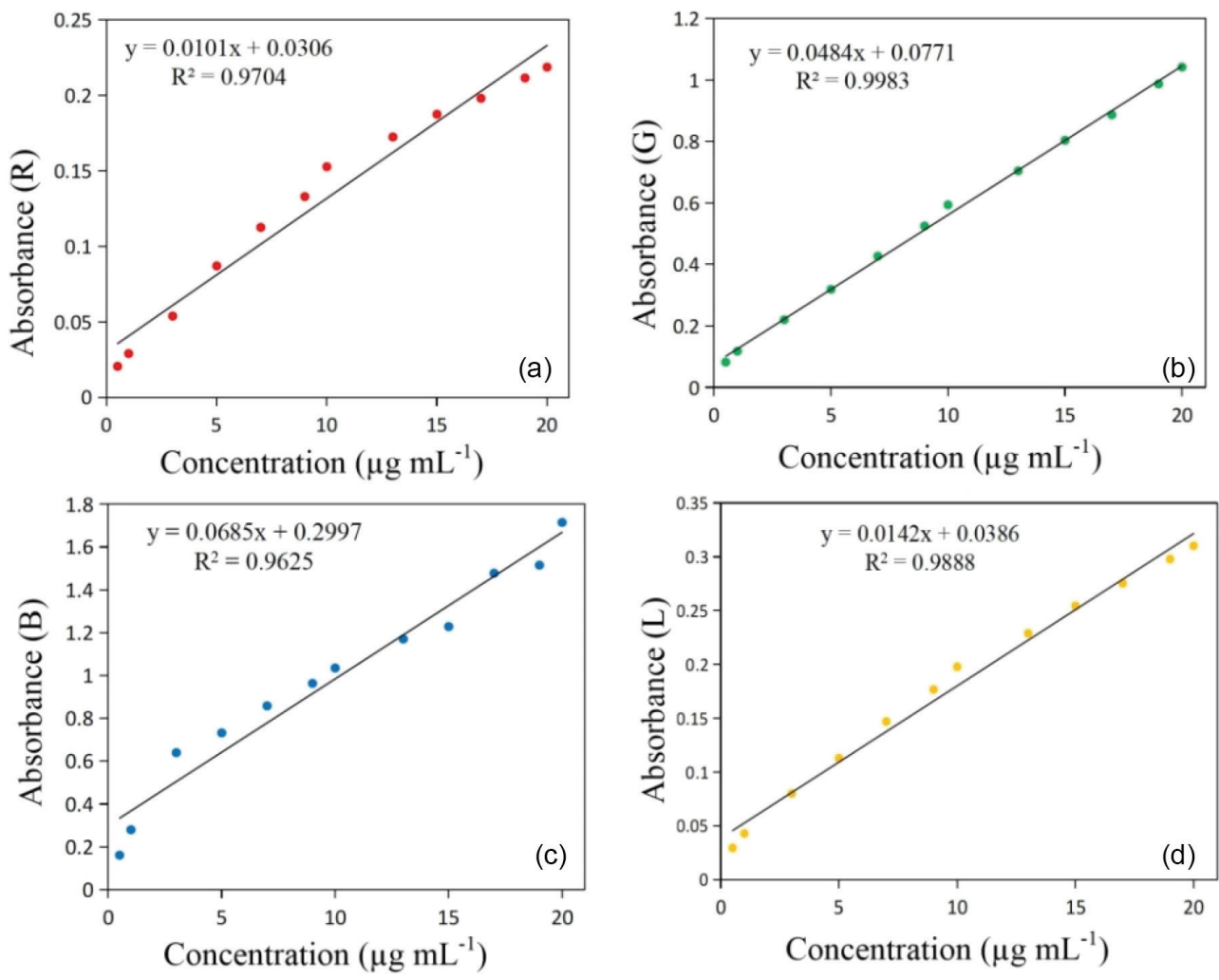

Figure 5. Fitting curve constructed by the absorbance value and vitamin C concentration using the: (a) red channel, (b) green channel, (c) blue channel, and (d) brightness channel

Figure $2 \mathrm{~S}(\mathrm{a})$ shows an exponential relationship between the grayscale value and vitamin $\mathrm{C}$ concentration, while Figure $2 \mathrm{~S}(\mathrm{~b})$ shows a linear relationship $(y=-0.0292 x+2.1702)$ constructed by using the log-grayscale value and vitamin $\mathrm{C}$ concentration, and the correlation coefficient $\mathrm{R}^{2}$ is 0.9767 . Nevertheless, the fitting effect of the $\mathrm{G}$ value and vitamin $\mathrm{C}$ concentration is better. The linear fitting of the color component and vitamin $\mathrm{C}$ concentration further improved the correlation of the fitting curve.

\section{Multivariate fitting results}

The experimental RGB and CIELab values were fitted with the vitamin $\mathrm{C}$ concentration using the multivariate function and both linear and nonlinear methods. The corresponding relationship expressions are shown in Table 3.

The correlation coefficient of the fitted curve obtained by the multivariate second-order polynomial fitting of the CIELab value and vitamin $\mathrm{C}$ concentration reaches 0.9994, and the fitting effect is best. The color components of the standard vitamin $\mathrm{C}$ solution collected by the image acquisition device and the analysis software were inputted into the multiple fitting curve to obtain the corresponding predicted concentration. A comparison of the predicted and actual vitamin $\mathrm{C}$ concentrations is shown in Figure 6.

The predicted curve of the multivariate data fit of the CIELab value to the vitamin $C$ concentration is consistent with the trend of the actual concentration value with a smaller error. The predicted curve obtained by fitting the multivariate second-order polynomial of the CIELab value to the vitamin $\mathrm{C}$ concentration is the closest to the actual concentration value.

Fitted models of the vitamin $\mathrm{C}$ concentration and the $\mathrm{R}, \mathrm{G}$, and CIELab values were obtained using exponential fitting, linear fitting, and multivariate second-order polynomial fitting, respectively. The three fitting methods were compared, and it was found that linear fitting improves the fitting effect. The predicted concentration of the multivariate second-order polynomial fitting of the CIELab values and the concentration multivariate were very close to the actual concentration, and the error was small. Multivariate fitting gave further play to the processing power of the computer and improved measurement accuracy. Therefore, the fitting curve between the CIELab values and the vitamin $\mathrm{C}$ concentration was chosen as the standard curve by fitting the second-order polynomial of multiple variables.

Table 3. Multivariate fitting expressions of the RGB and CIELab values with the vitamin C concentration

\begin{tabular}{lcc}
\hline Fitting method & Expression & Adj. R \\
RGB linear fitting & $y=0.2142 x_{1}-0.3083 x_{2}+0.1383 x_{3}$ & 0.9673 \\
RGB first-order polynomial & $y=-1.2624 x_{1}+0.4489 x_{2}-0.0865 x_{3}+141.1762$ & 0.9890 \\
RGB second-order polynomial & $y=0.0133 x_{1}{ }^{2}-3.2828 x_{1}-0.0011 x_{2}{ }^{2}-0.0297 x_{2}+0.0005 x_{3}{ }^{2}-0.0598 x_{3}+217.5101$ & 0.9980 \\
Lab linear fitting & $y=0.0309 x_{1}+0.4507 x_{2}+0.0347 x_{3}$ & 0.9962 \\
Lab first-order polynomial & $y=0.5089 x_{1}+0.8016 x_{2}+0.2156 x_{3}-40.5963$ & 0.9991 \\
Lab second-order polynomial & $y=0.0039 x_{1}{ }^{2}+0.2490 x_{1}+0.0071 x_{2}{ }^{2}+0.0252 x_{2}+0.0037 x_{3}{ }^{2}-0.2192 x_{3}+9.1526$ & 0.9994 \\
\hline
\end{tabular}



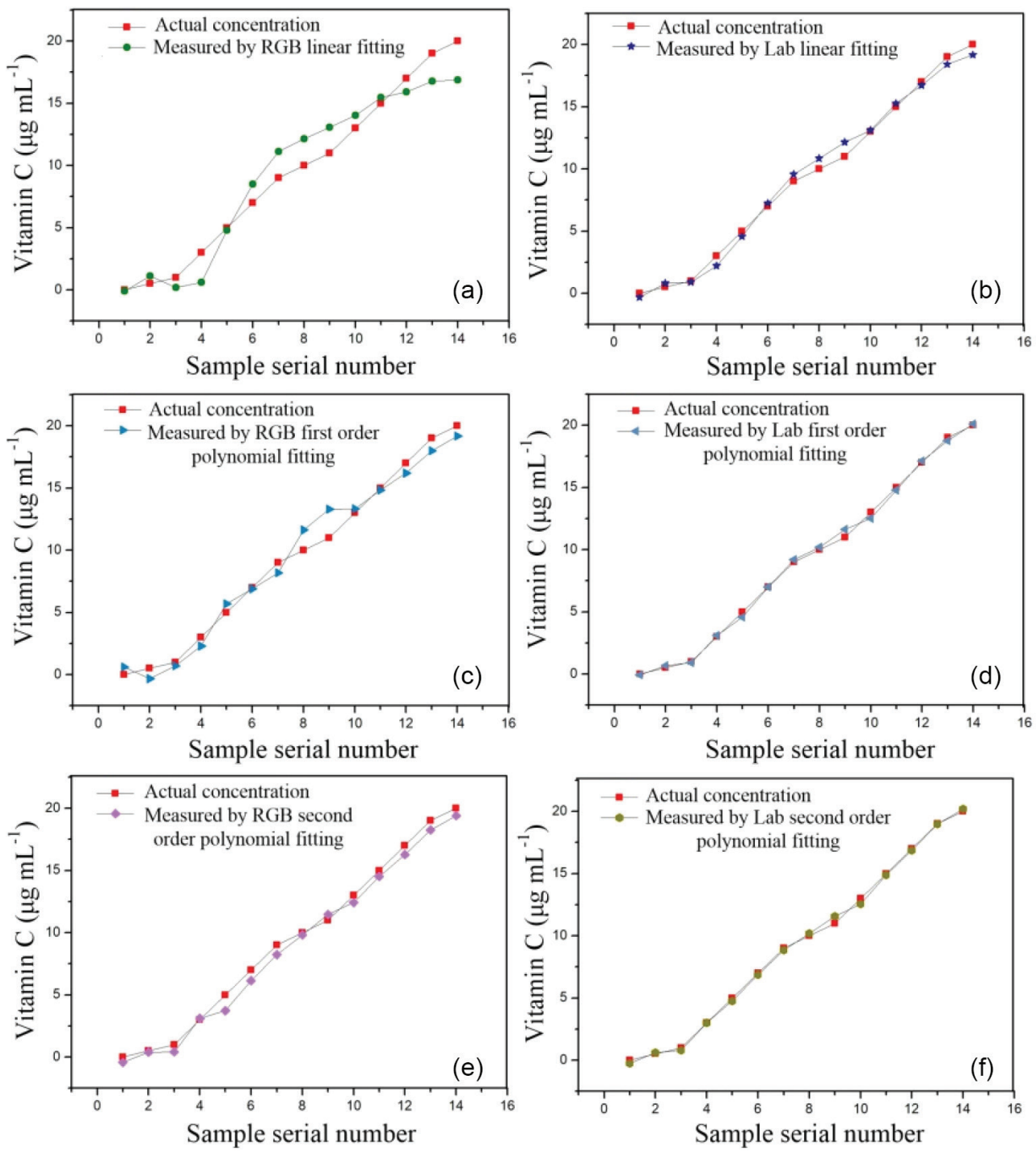

Figure 6. Comparison between the actual and predicted RGB and Lab predicted concentrations of vitamin $C$ using different fitting methods: (a)-(b) multiple linear fitting, $(c)-(d)$ multiple first-order polynomial fitting, and $(e)-(f)$ multiple second-order polynomial fitting

\section{$L O D, L O Q$, and measurement results of the standard vitamin $C$} solution

The limit of detection (LOD) refers to the smallest analyte (the analyzed sample component) concentration or mass that can be detected or quantified with a specified degree of certainty. ${ }^{37}$ The limit of quantification (LOQ) refers to the smallest analyte concentration or mass that can be quantitatively analyzed with reasonable reliability by a given procedure. ${ }^{38}$ According to the International Union of Pure and Applied Chemistry regulation, the LOD and LOQ can be calculated using formula (7).

$$
L O D=3 S D / k \quad L O Q=10 S D / k
$$

where SD is the standard deviation of the measured signal values of the blank samples $(n=10)$, and $\mathrm{k}$ is the standard curve slope. The concentration of vitamin $\mathrm{C}$ was fitted with the $\mathrm{Lab}, \mathrm{G}$, grayscale, and log-grayscale values to obtain the adjusted $\mathrm{R}^{2}$, LOD, and LOQ. The aforementioned values and vitamin $\mathrm{C}$ concentration ranges are listed in Table 4.

All values provided good fits, with the Lab value providing the best one. All LODs were below $0.5 \mu \mathrm{g} \mathrm{mL} \mathrm{m}^{-1}$, with the Lab values having the lowest one, facilitating the analysis of samples containing very low concentrations levels.
Table 4. The adjusted $\mathrm{R}^{2}$, LOD, $\mathrm{LOQ}$, and vitamin $\mathrm{C}$ concentration range values

\begin{tabular}{lcccc}
\hline Figures of Merit & Lab & $\mathrm{G}$ & Grayscale & Log-grayscale \\
\hline $\mathrm{R}^{2}$ & 0.9994 & 0.9983 & 0.9767 & 0.9767 \\
$\mathrm{LOD}(\mu \mathrm{g} \mathrm{mL}-1)$ & 0.24 & 0.28 & 0.30 & 0.30 \\
LOQ $\left(\mu \mathrm{g} \mathrm{mL}^{-1}\right)$ & 0.81 & 0.94 & 1.56 & 1.56 \\
Range $\left(\mu \mathrm{g} \mathrm{mL}^{-1}\right)$ & $0.81-20.00$ & $0.94-20.00$ & $1.56-20.00$ & $1.56-20.00$ \\
\hline
\end{tabular}

\section{Contrast with other methods}

Comparative experiment versus the standard method

To verify the accuracy of the proposed method, an experiment was designed to compare the obtained results with those of a spectrophotometer. The spectrophotometer used in this comparative experiment is a typical visible spectrophotometer (model $721 \mathrm{G}$, INESA Analytical Instrument Co., Ltd., Shanghai, China, http://www. inesa-instrument.com). The absorbance of different concentrations of the vitamin C standard solution was measured at the $475-\mathrm{nm}$ wavelength. Linear fitting of the absorbance determined by the spectrophotometer with different concentrations of the vitamin $\mathrm{C}$ 
Table 5. Partial test results of the concentration of the standard vitamin C solution using different methods

\begin{tabular}{lccccccccccc}
\hline Method & \multicolumn{8}{c}{ Vitamin C $\left(\mu \mathrm{g} \mathrm{mL} \mathrm{m}^{-1}\right)$} & \multicolumn{1}{c}{$\begin{array}{c}\text { Standard } \\
\text { deviation }\end{array}$} \\
Standard solution & 1 & 7 & 9 & 10 & 11 & 13 & 17 & 19 & 20 & 19 \\
RGB multivariate fitting & 0.831 & 6.305 & 8.838 & 9.620 & 10.585 & 13.348 & 16.591 & 18.526 & 19.583 & 9.693 \\
Lab multivariate fitting & 1.242 & 6.977 & 9.461 & 10.029 & 10.986 & 13.681 & 16.830 & 18.956 & 19.978 & 0.876 \\
Spectrophotometry & 1.190 & 6.948 & 9.912 & 10.682 & 11.903 & 14.491 & 17.652 & 18.405 & 19.880 & 2.273 \\
\hline
\end{tabular}

standard solution was carried out to obtain the standard curve, shown in Figure 3S.

The standard curve is $\mathrm{y}=0.1221 \mathrm{x}+0.0287$ with an $\mathrm{R}^{2}$ of 0.9968 . In the concentration range of the $0-20 \mu \mathrm{g} \mathrm{mL}^{-1}$ vitamin $\mathrm{C}$ solution, the concentration of vitamin $\mathrm{C}$ has a good linear relationship with the absorbance, which ensures the measurement accuracy in subsequent comparative experiments. The standard vitamin $\mathrm{C}$ solutions of different concentrations were determined by both the spectrophotometric and digital camera methods. Partial comparison results are presented in Table 5 .

Compared with the results of the spectrophotometric method, the multivariate fitting method of the CIELab values and concentration using the digital camera method had a smaller standard deviation and higher accuracy. The accuracy of the multivariate fitting method was $98.95 \%$, and the precision was determined with an RSD of $0.73 \%$. This method was shown to be the best for determining the vitamin $\mathrm{C}$ content in fruits and vegetables. Partial comparative results obtained by this method and the spectrophotometric method are shown in Figure 7.

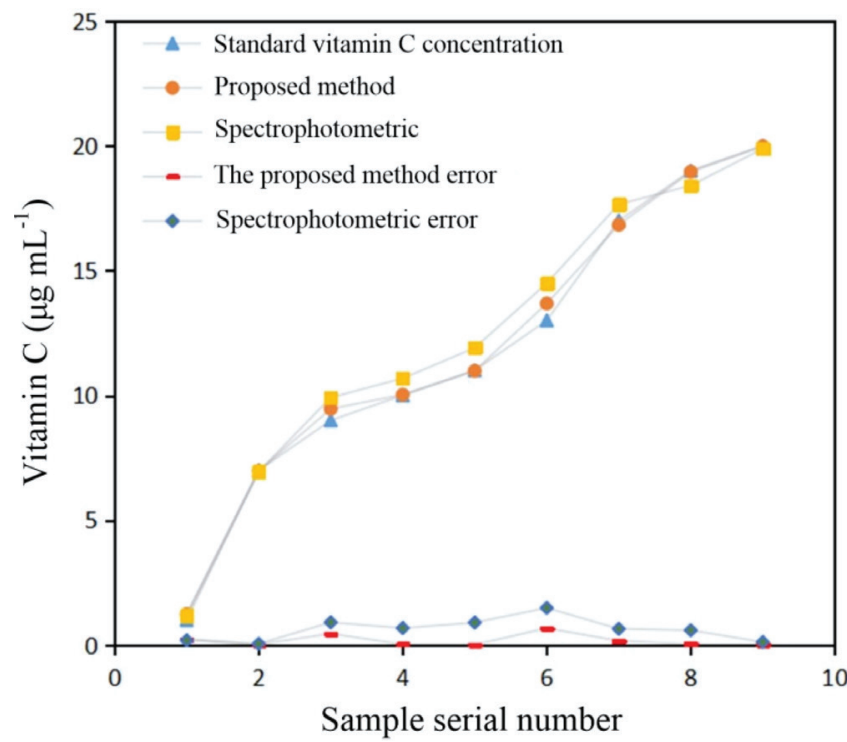

Figure 7. Comparison results of the spectrophotometric methods versus the proposed method

It can be seen that the trend of vitamin C concentration measured by the proposed method is consistent with the standard concentration, has a high degree of coincidence, and the measurement error is smaller than that measured by the spectrophotometric method. With the help of the powerful image and data processing technologies, the instrument design is simplified and the measurement accuracy is improved. Further, the measurement accuracy is improved through multilinear fitting, which combines the multiple color components and reduces random error.

\section{Determination of vitamin $\mathrm{C}$ content in fruits and vegetables}

To verify the practicability of the design, the proposed method and a $721 \mathrm{G}$ visible spectrophotometer were used to determine the content of vitamin $\mathrm{C}$ in several typical fruit and vegetable samples.

\section{Extraction of vitamin $C$}

The national standard method (i.e., GB method) was used to extract vitamin C. ${ }^{39}$ The edible parts of fruits or vegetables were weighed at $40 \mathrm{~g}$ and put into a homogenizer. Then, $40 \mathrm{~g}$ of $1 \%$ oxalic acid extract solution was added, and the mixture was quickly mashed into a homogenate. A sample of the homogenate was accurately weighed (10-40 g, accurate to $0.01 \mathrm{~g}$ ) in a beaker and then transferred to a $100 \mathrm{~mL}$ volumetric flask with $1 \%$ oxalic acid extract solution. The solution was diluted to scale, shaken well, and filtered. If the filtrate was colored, $0.4 \mathrm{~g}$ of kaolin was added for each gram of the sample for decolorization. Subsequently, the filtrate with kaolin was re-filtered to eliminate the interference of the kaolin color with the color of the fruit and vegetable solution. Finally, $15 \mathrm{~mL}$ of filtrate was placed into a centrifugal tube, and the filtrate was centrifuged for $5 \mathrm{~min}$ at 4,000 $\mathrm{r} \mathrm{min}^{-1}$; then, the samples of vitamin $\mathrm{C}$ for different fruits and vegetables were obtained.

\section{Determination of vitamin $C$}

First, the proposed method was used to determine the vitamin $\mathrm{C}$ content of commercially available tablets to calibrate the sample standard. In the actual measurement of the vitamin $\mathrm{C}$ content, $8.8 \mathrm{~mL}$ of the supernatant of each sample extracted from the fruits and vegetables was added to a $15-\mathrm{mL}$ test tube. Then, $0.6 \mathrm{~mL}$ of the $4.0 \mathrm{mg} \mathrm{mL}^{-1} \mathrm{o}$-phenanthrene solution and $0.6 \mathrm{~mL}$ of the $2.4 \mathrm{mg} \mathrm{mL}^{-1}$ ferric ammonium sulfate solution were added. After the sample solution was stable, the solution was placed in a colorimetric dish. The colorimetric dish was placed in a designed image acquisition device to obtain the image of the reaction solution. The RGB and CIELab values of the solution were obtained by the image processing software. The color information of several samples is shown in Figure 4S.

After the chromogenic reaction, the color information of some fruit and vegetable samples was collected by the digital camera, and the corresponding RGB and CIELab values were obtained. Under the same conditions, the content of the above vitamin $\mathrm{C}$ samples was determined by spectrophotometry and compared to the content determined by the proposed method to verify accuracy.

\section{Interference and recovery assays}

Foreign substances such as sugar, amino acids, vitamins, and reducing acids were added to the standard $9 \mu \mathrm{g} \mathrm{mL}^{-1}$ vitamin $\mathrm{C}$ solution to verify whether these substances affected the measurement results of vitamin C. Moreover, a recovery experiment was carried out to ensure that the matrix effect did not affect the results. Table 6 lists the vitamin $\mathrm{C}$ concentrations determined after the addition of different concentrations of foreign substances.

The results indicated that the addition of citric acid, glucose, vitamin $\mathrm{E}$, and tryptophan at any concentration did not affect the determination of vitamin $\mathrm{C}$ content. However, when sulfite was added into the solution in a ratio of $1: 10$, the vitamin $C$ concentration was slightly higher. However, the relative error of 
Table 6. Potential interferents for vitamin $\mathrm{C}$ determination

\begin{tabular}{cccccc}
\hline & \multicolumn{2}{c}{ Potential interferentes / (\%) } \\
\hline $\begin{array}{c}\text { Analyte: Interference } \\
\text { ratio }\end{array}$ & Citric acid & Glucose & Vitamin E & Sulfite & Tryptophan \\
\hline $1: 0.1$ & 0.17 & 0.49 & -0.18 & 1.59 & 0.64 \\
$1: 1$ & 0.96 & 0.63 & -0.39 & 2.82 & 2.04 \\
$1: 10$ & 1.06 & 0.90 & -0.36 & 2.00 & 4.09 \\
\hline
\end{tabular}

the vitamin $\mathrm{C}$ concentration did not exceed 5\%, and the content of sulfite in the actual fruit and vegetable samples was low; thus, it was confirmed that these substances did not interfere with the experimental results.

To verify the impact of the matrix effect in fruits and vegetables, the relative recovery of different samples was obtained, and the absolute recovery was calculated to obtain the extraction efficiency. Table 7 summarizes the experimental results for different fruits and vegetables.

The results of the recovery experiments of different samples showed that the relative recovery rate was $94.3 \%-108.9 \%$, indicating that the matrix effect in different samples had no significant influence on vitamin $\mathrm{C}$ content. The absolute recovery rate is $64.3 \%-84.8 \%$; therefore, the experimental results are considered satisfactory.

\section{Determination and comparison of actual samples}

The content of vitamin $\mathrm{C}$ in fruit and vegetable samples was determined by both the proposed and spectrophotometric methods; the results are summarized in Table 8. SPSS software was used to test the results of the two methods using the Student's t-test and variance ratio F-test.

The error difference between the proposed and spectrophotometric methods is small. The results were statistically evaluated in terms of the Student's t-test and variance ratio F-test, and the values calculated were found to be less than the tabulated values at the $95 \%$ confidence level, indicating no significant differences in the accuracy and precision between the two methods. Additionally, the results acquired by the proposed method follow the same trend as those of the spectrophotometric method.

\section{CONCLUSIONS}

In this study, a method of measuring vitamin $\mathrm{C}$ concentration using a digital camera and organic compound color reaction was proposed. The RGB and CIELab values of the solution were obtained by developing a measurement device and implementing image processing software. The relationship between various color components and vitamin $\mathrm{C}$ concentration was determined, and the corresponding curves were constructed using nonlinear, linear, and multivariate fitting. The relationship curve constructed by multivariate fitting enhances computer processing, optimizes the fitting effect, and improves the measurement accuracy. The content of vitamin $\mathrm{C}$ in the standard solution and actual fruits and vegetables was determined by the proposed method and compared with that obtained by the spectrophotometric method. The comparison results show that the method of the digital camera combined with the color reaction of colored compounds is more accurate than the spectrophotometric method. During the determination of the vitamin $\mathrm{C}$ content in real fruits and vegetables, the results of an independent sample t-test showed that there was no significant difference between the proposed method and the spectrophotometric method. Furthermore, the proposed method has the advantage of high accuracy and simple instrumentation design. Therefore, it can replace the traditional spectrophotometric method for the measurement of vitamin $\mathrm{C}$ concentration in fruits and vegetables while reducing the measurement cost.

\section{SUPPLEMENTARY MATERIAL}

We will provide readers with the datasheet of the backlight board circuit components, camera acquisition source program of the

Table 7. Results of different sample recovery experiments

\begin{tabular}{|c|c|c|c|}
\hline Sample & Vitamin $C$ test value $\left(\mu \mathrm{g} \mathrm{mL^{-1 } )}\right.$ & Relative recovery & Absolute recovery \\
\hline Tomato & 4.96 & $108.9 \%$ & $64.3 \%$ \\
\hline Chinese cabbage & 3.01 & $95.4 \%$ & $73.5 \%$ \\
\hline Kiwi fruit & 6.82 & $102.8 \%$ & $84.8 \%$ \\
\hline Lemon & 4.49 & $95.6 \%$ & $84.4 \%$ \\
\hline Cucumber & 1.80 & $94.3 \%$ & $71.3 \%$ \\
\hline
\end{tabular}

Table 8. The results of the proposed and spectrophotometric methods for the determination of the vitamin C content in actual fruits and vegetables

\begin{tabular}{|c|c|c|c|c|}
\hline \multirow{2}{*}{ Fruit/vegetable } & \multicolumn{2}{|c|}{ Concentration $\left(\mu \mathrm{g} \mathrm{mL}^{-1}\right)$} & \multirow{2}{*}{$t$-test $\mathrm{t}^{\mathrm{b}}$} & \multirow{2}{*}{$F$-test $^{\mathrm{c}}$} \\
\hline & Proposed method $^{\mathrm{a}}$ & Spectrophotometric method ${ }^{a}$ & & \\
\hline Cucumber & $2.660 \pm 0.022$ & $2.664 \pm 0.079$ & 0.097 & 1.615 \\
\hline Apple & $3.08 \pm 0.294$ & $3.25 \pm 0.136$ & 2.449 & 4.741 \\
\hline Peach & $0.940 \pm 0.018$ & $0.993 \pm 0.083$ & 1.244 & 1.746 \\
\hline Tomato & $2.01 \pm 0.014$ & $1.96 \pm 0.058$ & 1.663 & 3.245 \\
\hline
\end{tabular}

${ }^{a}$ Mean \pm standard deviation $(n=5)$. ${ }^{\text {T}}$ Tabulated $t$-value for four degrees of freedom at $\mathrm{P}(0.95)$ is 2.78 . ${ }^{\mathrm{c}}$ Tabulated F-value for $(4,4)$ degrees of freedom at $\mathrm{P}$ $(0.95)$ is 6.39 . 
program written in C\# Language, third-party component Camera_Net Library used for programming, and corresponding development platform. Some images of the systems used in this work are available at https://www.quimicanova.sbq.org.br/ in the form of a PDF file, with free access.

\section{ACKNOWLEDGMENTS}

This work is partially supported by the National Natural Science Foundation of China (no. 61671434) and the Natural Science Fund for Colleges and Universities of Anhui Province (No. KJ2017ZD32).

\section{REFERENCES}

1. Carr, A. C.; Maggini, S.; Nutrients 2017, 9, 1211.

2. Straaten, H. M. O.; Man, A. M. S.; Waard, M. C. D.; Crit. Care 2014, 18,1 .

3. Nishikimi, M.; Yagi, K.; Subcell. Biochem. 1996, 25, 17.

4. Padayatty, S. J.; Katz, A.; Wang, Y.; Eck, P.; Kwon, O.; Lee, J.; Chen, S.; Corpe, C.; Dutta, A.; Dutta, S. K.; Levine, M.; J. Am. Coll. Nutr. 2003, $22,18$.

5. Kallner, A.; Annu. N. Y. Acad. Sci. 1987, 498, 418.

6. Lamarche, J.; Nair, R.; Peguero, A.; Courville, C.; Int. J. Nephrol. 2011, 2011, 1 .

7. Zhou, S. S.; Zhou, Y.; World J. Diabetes 2014, 5, 1.

8. Mussa, S. B.; Sharaa, I. E.; J. Appl. Phys. 2014, 6, 46.

9. Lu, Q.; Chen, X.; Liu, D.; Wu, C.; Liu, M.; Li, H.; Zhang, Y.; Yao, S.; Microchim. Acta 2019, 186, 72.

10. Russell, L. F.; J. Food Sci. 1986, 51, 2.

11. Barberis, A.; Bazzu, G.; Calia, G.; Puggioni, G.; Rocchitta, G.; Migheli, R.; Schirra, M.; Desole, M. S.; Serra, P. A.; Anal. Chem. 2010, 82, 5134.

12. Nweze, C. C.; Abdulganiyu, M. G.; Erhabor, O. G.; Int. J. Sci. Environ. Technol. 2015, 4, 17.

13. Kabasakalis, V.; Siopidou, D.; Moshatou, E.; Food Chem. 2000, 70, 325.

14. Spínola, V.; Mendes, B.; Câmara, J. B.; Castilho, P. C.; LWT--Food Sci. Technol. 2013, 50, 489.

15. Arya, S. P.; Mahajan, M.; Jain, P.; Anal. Sci. 1998, 14, 889.
16. Pathy, K. S.; Surgery \& Case Studies 2018, 1, 52.

17. Kul, D.; Ghica, M. E.; Pauliukaite, R.; Brett, C. M.; Talanta 2013, 111, 76.

18. Pisoschi, A. M.; Pop, A.; Serban, A. I.; Fafaneata, C.; Electrochim. Acta 2014, 121, 443 .

19. de Oliveira, L. M. A.; dos Santos, V. B.; da Silva, E. K. N.; Lopes, A. S.; Dantas-Filho, H. A.; Talanta 2020, 206, 120219.

20. Soares, S; Rocha, F. R. P; Talanta 2019, 199, 285.

21. Thajee, K.; Paengnakorn, P.; Wongwilai,W.; Grudpan, K.; Talanta 2018, $185,160$.

22. Choodum, A.; Kanatharana, P.; Wongniramaikul, W.; Daéid, N. N.; Talanta 2013, 115, 143.

23. Coutinho, M. S.; Morais, C. L. M.; Neves, A. C. O.; Menezes F. G.; Lima, K. M. G.; J. Braz. Chem. Soc. 2017, 28, 2500

24. dos Santos V. B.; da Silva, E. K. N.; de Oliveira, L. M. A.; Suarez, W. T.; Food Chem. 2019, 285, 340.

25. Kolthoff, I. M.; Leussing, D. L.; Lee, T. S.; J. Am. Chem. Soc. 1951, 73, 390.

26. Seid, M. A.; Leung, S. H.; Sharpe Elles, L. M.; Shaver L. A.; J. Chem. Educ. 2016, 93, 1766.

27. Gardner, C. M.; J. Biomed. Opt. 2018, 23, 018001.

28. Baldevbhai, P. J.; Anand, R. S.; J. Electron. Commun. Eng. 2012, 1, 24.

29. Chung, K. L.; Yang, W. N.; Lai, Y. R.; Lin, L. C.; Signal Image Video P. 2014, 8, 1691.

30. Murata, H.; Saitoh, K.; Sumida, Y.; J. Meteorol. Soc. Jpn. 2018, 96B, 211

31. Wu, Z.; Xu, E.; Long, J.; Wang, F.; Xu, X.; Jin, Z.; Jiao, A.; Food Control 2015, 56, 95.

32. Ma, X. Y.; Zhang, Y. B.; Cao, H.; Zhang, S. L.; Zhou, Y.; J. Spectrosc. 2018, 2018, 1 .

33. Munmai, A.; Somsook, E.; Chem. Educ. 2011, 16, 323

34. Leeuw, T.; Boss, E.; Sensors 2018, 18, 256.

35. Lee, T. H.; Kim, B. K.; Song, W. J.; Opt. Eng. 2010, 49, 057006.

36. Kanan, C.; Cottrell, G. W.; PLoS One 2012, 7, e29740.

27. Fassel, V.; Spectrochim. Acta B 1978, 33, 241.

28. MacDougall, D.; Crummett, W. B.; Anal. Chem. 1980, 52, 2242.

39. Oroian, M.; Escriche, I.; Food Res. Int. 2015, 74, 10. 